\title{
DESIGN AND IMPLEMENTATION OF DUAL-STAGE TRACK-FOLLOWING CONTROL FOR HARD DISK DRIVES
}

\author{
Jianbin Nie \\ Computer Mechanics Laboratory \\ Department of Mechanical Engineering \\ University of California, Berkeley \\ Berkeley, CA 94720 \\ njbin@berkeley.edu
}

\author{
Roberto Horowitz \\ Professor of Mechanical Engineering \\ University of California, Berkeley \\ Berkeley, CA 94720 \\ horowitz@berkeley.edu
}

\begin{abstract}
This paper discusses the design and implementation of two track-following controllers for dual-stage hard disk drive servo systems. The first controller is designed by combining an outer loop sensitivity-decoupling (SD) controller with an inner loop disturbance observer (DOB). The second is designed by combining mixed $\mathrm{H}_{2} / \mathrm{H}_{\infty}$ synthesis techniques with an add-on integral action. The designed controllers were implemented and evaluated on a disk drive with a PZT-actuated suspension-based dual-stage servo system. Position error signal (PES) for the servo system was obtained by measuring the slider displacement with an LDV and injecting a simulated track runout.
\end{abstract}

\section{INTRODUCTION}

The continuously increasing storage capacity of hard disk drives (HDD) poses a great challenge to precisely position the $\mathrm{read} / \mathrm{write}$ head on the desired track. Typically, tracking performance is measured by track mis-registration (TMR). The TMR can be broken down into a component due to repeatable runout (RRO) and a component due to non-repeatable runout (NRRO). RRO mainly results from disk eccentricity, non-ideal servo track writing [1], and spindle motor vibration and is hence synchronous with the disk rotation speed. All other runout other than RRO is referred to as NRRO. Furthermore, non-repeatable runout can be in turn categorized into torque disturbance, windage, non-repeatable disk motions and measurement noises. The torque disturbance, which is mainly caused by the bias force of the flexible cable, the pivot friction and the air-turbulence impinging on the voice coil motor (VCM), is typically a low frequency disturbance. Windage, which is mainly due to air-turbulence directly exciting suspension resonance modes, is primarily a high frequency disturbance. Non-repeatable disk motions, which directly affect the position of $\mathrm{R} / \mathrm{W}$ head relative to the servo track, lead to additional track runout. Measurement noise, representing the effects of PES demodulation noise, includes electrical noise and A/D quantization noise. The goal of the HDD servo system is to reduce TMR as much as possible. A great deal of research effort has been focused on the development of disturbance rejection algorithms for minimizing RRO, such as adaptive feedforward cancellation (AFC) [2-3] and this topic will not be pursued in this paper.

Dual-stage actuation (DSA), which combines the traditional VCM with an additional microactuator (MA), has been proposed as a means of enhancing servo tracking performance by increasing the servo bandwidth. Several dual-stage control design methods have been studied for NRRO rejection. And DSA track-following control design can be roughly classified into single-input-single-output classical loop-shaping design techniques, such as sensitivity-decoupling control [4] and the PQ method [5], and multivariable optimal control design, such as LQG [6], $\mathrm{H}_{\infty}$ [7] and $\mu$-synthesis [8].

In this paper, we present two track-following control designs for dual-stage servo systems. The first controller was designed by combining an outer loop sensitivity-decoupling controller with an inner loop disturbance observer [9]. Two different $Q$ filters were utilized in the inner-loop disturbance observer to achieve both good disturbance rejection and robust stability, by considering that the plant uncertainties for the $\mathrm{VCM}$ and the microactuator are quite different. The second one was designed by using a mixed $\mathrm{H}_{2} / \mathrm{H}_{\infty}$ control synthesis methodology in order to minimize a nominal $\mathrm{H}_{2}$ norm for a good performance as well as to satisfy an $\mathrm{H}_{\infty}$ constraint for the robust stability. The conservatism of the mixed $\mathrm{H}_{2} / \mathrm{H}_{\infty}$ control via a convex optimization with linear matrix inequalities (LMI) was discussed and a nominal $\mathrm{H}_{2}$ controller was designed by tuning control input weighting values to achieve the robustness. 
In addition, an add-on integral action was incorporated to cancel constant and low-frequency disturbances. The designed controllers were implemented on a PZT-actuated suspension dual-stage servo system, which utilizes the microactuator to bend the suspension to generate a controlled fine radial head motion. The servo system's PES was obtained from the output of a laser Doppler vibrometer (LDV), which measures the absolute radial slider displacement. Since such a setup does not have track runout, a computer generated runout signal was injected into the control system to simulate track motion.

The paper is organized as follows. Section 2 describes our test setup and the corresponding system identification. In Section 3, the two control designs are presented. Implementation results are shown in Section 4. The conclusion is given in Section 5 .

\section{TEST SETUP AND MODELING OF THE DUAL-STAGE SERVO SYSTEM}

\subsection{Disturbance observer design}

Figure 1 shows a picture of the experimental setup. A PZT-actuated suspension was assembled to an arm of the E-block of a commercial 3.5" 7200-rpm disk drive. An LDV was utilized to measure the absolute radial displacement of the slider. The resolution of the LDV is $2 \mathrm{~nm}$ for the measurement gain of $0.5 \mu \mathrm{m} / \mathrm{V}$. The control circuits include a Texas Instrument TMS320C6713 DSP board and an in-house made analog board with a 12-bit ADC, a 12-bit DAC, a voltage amplifier to drive the MA, and a current amplifier to drive the VCM. The DSP sampling frequency is $71.4 \mathrm{KHz}$ in this paper. And the input delay including $\mathrm{ADC}$ and $\mathrm{DAC}$ conversion delay and DSP computation delay is $6 \mu \mathrm{s}$. A hole was cut through the case of the drive to make laser go into the drive. It should be noted that these modifications affected the response of the drive and may have detrimentally affected the attainable performance of the servo system, as discussed in Section 4.

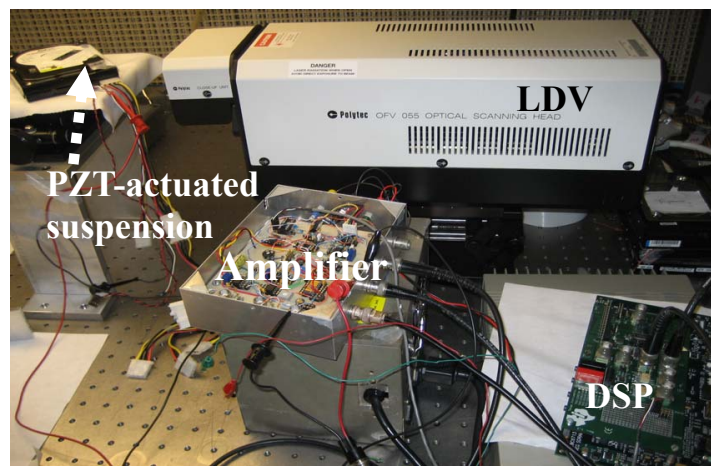

FIGURE 1. EXPERIMENT SETUP

\subsection{Dual-stage actuator modeling}

The frequency response of the VCM shown in Fig. 2, was measured from the input to its current amplifier to the slider motion, while the frequency response of the microactuaor shown in Fig. 3, was measured from the input to its voltage amplifier to the slider motion. The experiment frequency responses show that the flexi-cable mode is around $160 \mathrm{~Hz}$ and the microactuator resonance mode is around $18 \mathrm{KHz}$. Then, we did frequency response fitting for the experiment frequency responses by using Weighted Least Square (WLS) techniques. Consequentially, the fitted VCM and micro-actuator transfer functions have 12 states and 10 states respectively.

The experiment frequency responses show that the VCM and the micro-actuator have some common suspension modes. To reduce the system order, all of the five suspension modes are treated as common modes. Then, the dual-stage actuator system was modeled as a double-input-single-output system with 12 states after the common mode identification. The simulated frequency responses for the identified VCM and MA models are shown in Fig. 2 and Fig. 3 respectively. With the identified disturbances presented in Section 2.3, the complete dual-stage servo system is modeled as the block diagram in Fig. 4.

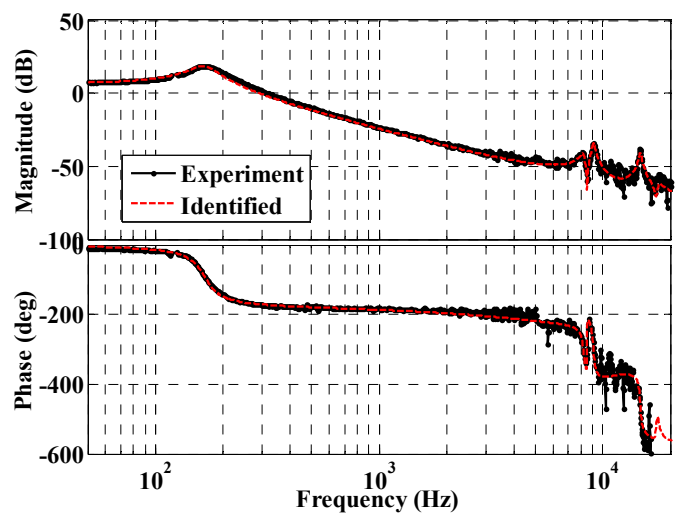

FIGURE 2. VCM FREQUENCY RESPONSE

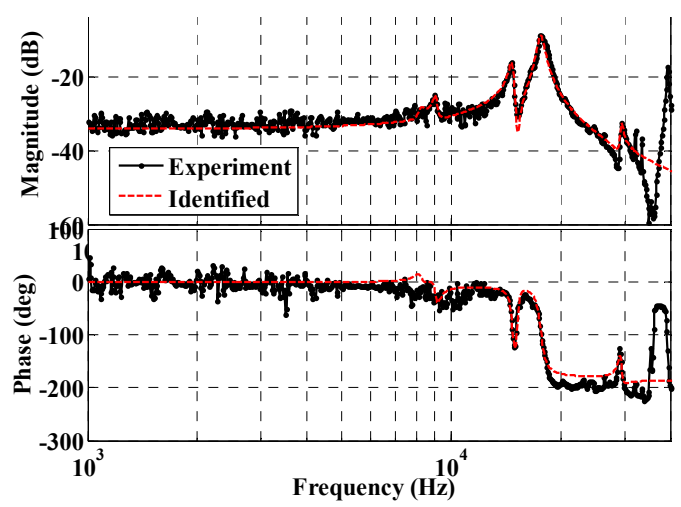

FIGURE 3. MICROACTUATOR FREQUENCY RESPONSE

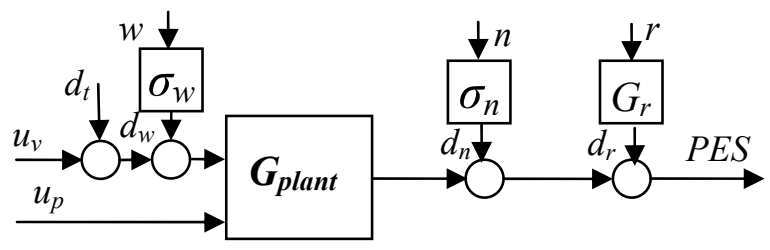

FIGURE 4. MODELING OF COMPLETE DSA SERVO 


\subsection{Disturbance identification}

2.3.1 Measurement noise $\left(\boldsymbol{d}_{\boldsymbol{n}}\right)$. As the LDV integrates velocity signals to calculate displacement signals, its displacement measurement has a low frequency drift, which can be seen from the LDV measurement noise power spectrum density (PSD) in Fig. 5. The low frequency measurement noise can be considered as runout and is captured by a second order low pass filter shown in Fig. 5. At high frequency, the LDV measurement noise was modeled as white noise with $\sigma_{n}=1.3$ $\mathrm{nm}$.

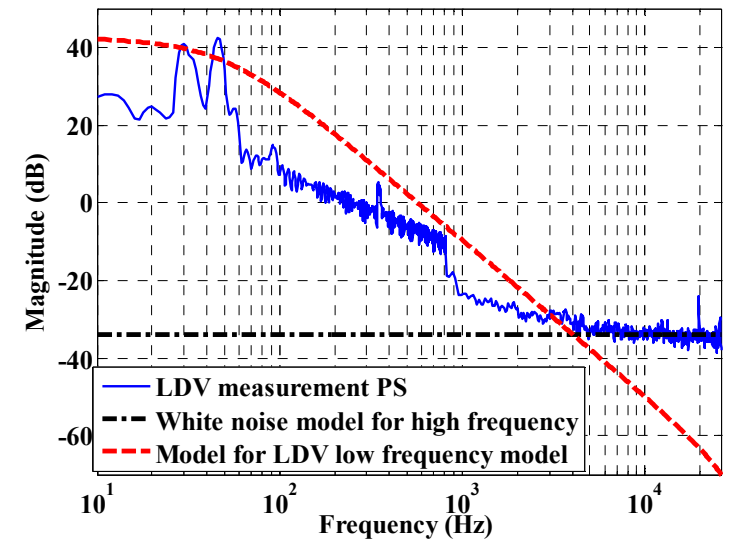

FIGURE 5. LDV NOISE POWER SPECTRUM DENSITY

2.3.2 Windage torque disturbance identification $\left(\boldsymbol{d}_{\boldsymbol{w}}\right)$. The windage torque disturbance, which is caused by the air-turbulence impinging on the VCM and directly exciting the suspension modes and is known to be a broad band excitation, is assumed to white and denoted by the input signal $d_{w}$ to VCM in Fig. 4. The amplitude $\sigma_{w}$ was estimated by matching the power spectrum density of the absolute open loop slider motion.

2.3.3 Runout identification $\left(d_{r}\right)$. Although there is no track runout for our experiment setup, a runout model, characterized from the track runout data of a real drive, was included to make our control design more realistic. The real track runout caused by disk vibrations has several disk modes between $1 \mathrm{KHz}$ and $3 \mathrm{KHz}$. In order to make the control synthesis simple, the peaks were characterized by a second order envelop shown in Fig. 6. Note that the LDV low frequency measurement noise is also treated as runout and the measurement noise is much higher than the real track runout at low frequency. Then, the LDV low frequency measurement noise and the second-order envelop for the disk modes were combined to construct the complete runout model shown in Fig. 6 . The root-mean-square (RMS) value of this runout model is $118.12 \mathrm{~nm}$

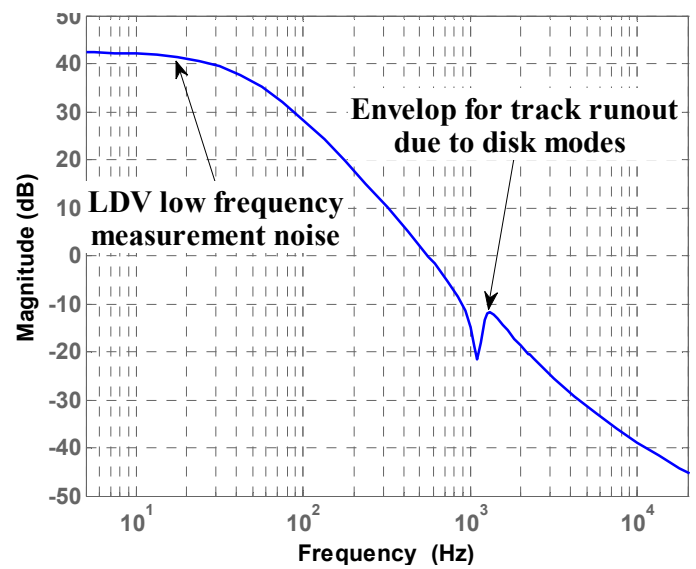

FIGURE 6. COMPLETE RUNOUT MODEL

2.3.4 Low frequency torque disturbance $\left(d_{t}\right)$. The torque disturbances due to the bias force of the flexible cable and the pivot friction have nonlinearities, which makes it difficult to model these disturbances. However, these torque disturbances are mainly at low frequency. Thus, to reject these torque disturbances, we just incorporated an integral action into the controllers instead of modeling these torque disturbances for our control design.

\section{DSA TRACK-FOLLOWING CONTROL DESIGN}

\subsection{Disturbance observer design}

3.1.1 Design methodology. Disturbance observer control has been broadly used in mechatronic systems to do disturbance rejection with a proper $Q$ filter selection [10]. In this paper, this technique was extended to dual-stage actuation servo systems. By considering that the VCM and the microactuator have different plant uncertainties, two different $Q$ filters were designed to achieve both good performance and stability. The sensitivity-decoupling dual-stage servo technique [4] was used to design the servo system's outer loop control. Figure 7 shows the block diagram of the disturbance observer, where $G_{v}{ }^{n}, G_{p}{ }^{n}$ and $d_{t o t}$ represent the nominal VCM plant, the nominal MA plant and the overall effect of all disturbances respectively. Note that the nominal plants should be minimum phase. Their magnitude bode plots are shown in Fig. 8

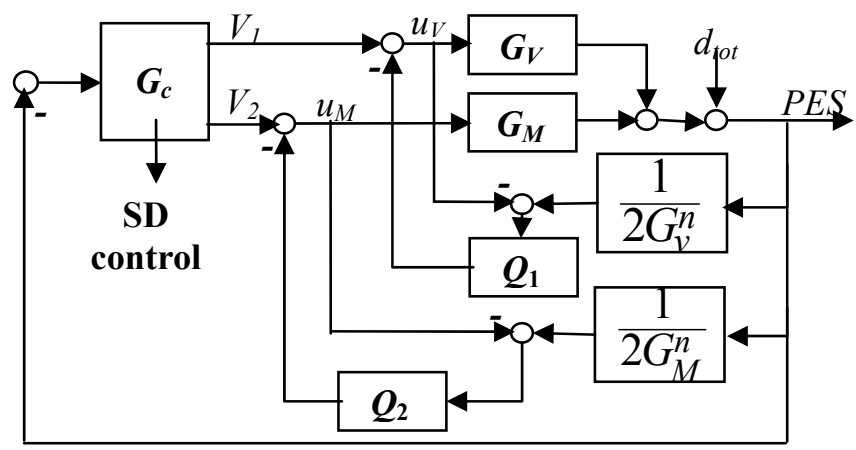

FIGURE 7. DUAL-STAGE DISTURBANCE OBSERVER 


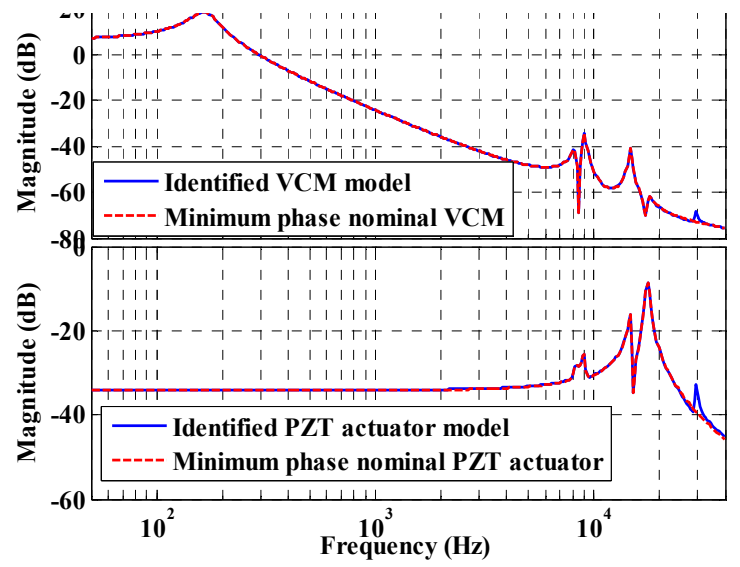

FIGURE 8. NOMINAL PLANTS FOR VCM AND MA

Then the PES can be written as:

$$
\operatorname{PES}(s)=\frac{\frac{1-Q_{2}}{1-Q_{1}} G_{V}(s) V_{1}(s)+G_{M}(s) V_{2}(s)+\left(1-Q_{2}(s)\right) d_{t o t}(s)}{\frac{1}{2}\left(1+Q_{2} \Delta_{2}(s)+\frac{1-Q_{2}}{1-Q_{1}}\left(1+Q_{1} \Delta_{1}(s)\right)\right)}
$$

where $\Delta_{1}$ and $\Delta_{2}$ are respectively the VCM and MA model uncertainties, i.e. $G_{V}(s)=G_{V}^{n}(s)\left(1+\Delta_{1}(s)\right) \quad$ and $G_{M}(s)=G_{M}^{n}(s)\left(1+\Delta_{2}(s)\right)$. For the robust stability, the $Q_{1}$ and $Q_{2}$ must satisfy the following constraint.

$$
\left\|\frac{\left(1-Q_{1}\right) Q_{2} \Delta_{2}+\left(1-Q_{2}\right) Q_{1} \Delta_{1}}{1-Q_{1}+1-Q_{2}}(s)\right\|_{\infty}<1
$$

By considering the arbitrariness of $\Delta_{1}$ and $\Delta_{2}$, the robust stability constraint is transformed to the following constraint.

$$
\frac{\left|\left(1-Q_{1}\right) Q_{2} \Delta_{2}(j \omega)\right|+\left|\left(1-Q_{2}\right) Q_{1} \Delta_{1}(j \omega)\right|}{\left|\left(1-Q_{1}+1-Q_{2}\right)(j \omega)\right|}<1 \text {, for all } \omega
$$

3.1.2 Selection of $\boldsymbol{Q}$ filters. Equation (1) shows that the disturbances rejection function of the servo system can be shaped by changing $Q_{1}(s)$ and $Q_{2}(s)$. In addition, the $Q$ filters must be designed to satisfy the robust stability requirement in Eq. (3). Since many of the largest disturbances are at low frequency, the selection of high-pass filters of $1-Q_{\mathrm{i}}(\mathrm{i}=2)$ facilitates the low frequency disturbance rejection. Meanwhile, such a selection makes $Q_{\mathrm{i}}(\mathrm{i}=2)$ be low-pass filters and thus facilitates the attainment of the robust stability as well, since plant uncertainties are significant at high frequency. Moreover, in order to follow the track runout due to disk motions, $Q_{2}$ was designed to achieve good attenuation at the $1 \mathrm{KHz}$ to $2 \mathrm{KHz}$ frequency range for the overall sensitivity function from $d_{t o t}$ to $P E S$ shown in Fig. 11. In addition, by considering that the microactuator has less uncertainty than the $\mathrm{VCM}$ at high frequency, we designed the $Q$ filters in such way that the DC gain of $Q_{2}$ for the microactuator is higher than that of $Q_{1}$ for the
VCM and the corner frequency of $Q_{2}$ is also higher than that of $Q_{1}$. In order to make our controller synthesis simple, the filters were chosen as first-order systems. Figure 9 shows the final selection of the $Q$ filters. In order to verify the attainment of robust stability constraint Eq. (3), the magnitude of its left term, shown in Fig. 10, was calculated by replacing $\Delta_{1}$ and $\Delta_{2}$ with the differences between the nominal plants and the experiment frequency responses.

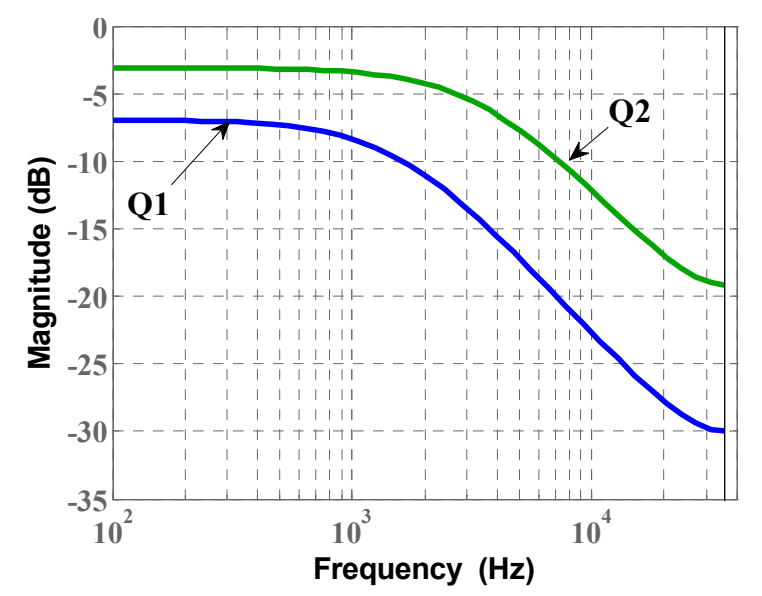

FIGURE 9. SELECTION OF Q FILTERS

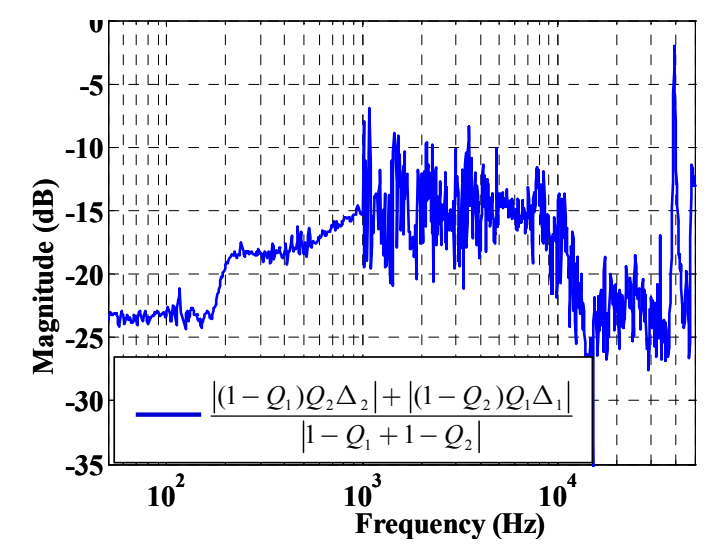

FIGURE 10. ROBUST STABILITY VERIFICATION

3.1.3 Outer loop control design. The outer loop controller was designed according to the standard sensitivity-decoupling synthesis technique [4]. The VCM loop controller was designed to have a PI controller with a lead compensator and a notch filter to attenuate the biggest resonance peak at $9.06 \mathrm{KHz}$. The resulting gain crossover frequency for the VCM loop sensitivity function is $587 \mathrm{~Hz}$. Since the microactuator has higher bandwidth than the VCM, the MA loop achieves a higher gain crossover frequency at 1.64 $\mathrm{KHz}$, by designing the MA loop controller as a lag compensator with three notch filters to attenuate the three resonance peaks at 14.70 KHz, $17.71 \mathrm{KHz}$ and $29.34 \mathrm{KHz}$. The corresponding design results are shown in Fig. 11. The resulting gain margin, phase margin and gain crossover frequency for the overall outer loop are $6.72 \mathrm{~dB}, 28.8^{\circ}$ and $1.34 \mathrm{KHz}$ respectively. 


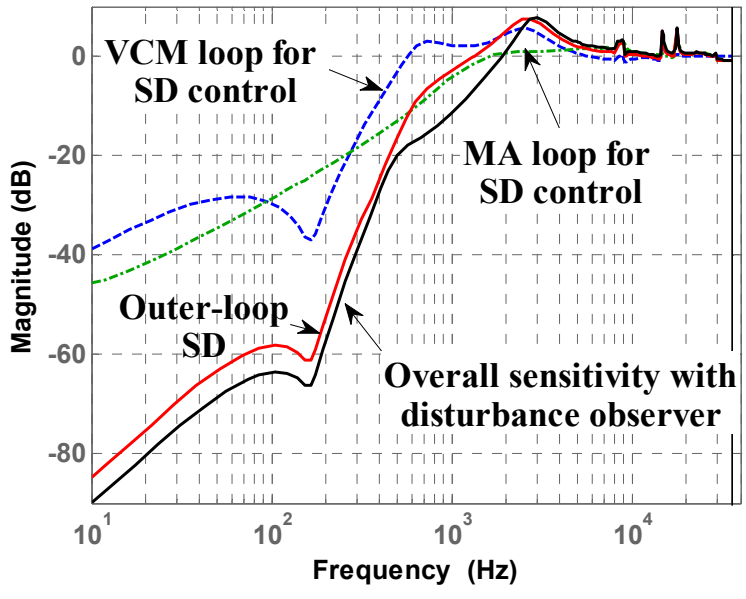

FIGURE 11. SENSITIVITY FUNCTION FOR DOB DESIGN

As shown in Fig. 11, by using the disturbance observer outlined in this section, the overall gain margin, phase margin and gain crossover frequency can be improved to be $6.83 \mathrm{~dB}$, $30.7^{\circ}$ and $2.08 \mathrm{KHz}$ respectively.

\subsection{Mixed $\mathrm{H}_{2} / \mathrm{H}_{\infty}$ design with add-on integral action}

3.2.1 Design methodology. In this section the dual-stage track-following servo synthesis problem is formulated by minimizing the variance of the PES while maintaining robust stability in the presence of plant input multiplicative unstructured uncertainties as described in Eq. (4).

$$
P(s)=P_{n}(s)\left(I_{2}+\left[\begin{array}{cc}
W_{\Delta V}(s) & 0 \\
0 & W_{\Delta M}(s)
\end{array}\right] \Delta(s)\right),\|\Delta(s)\|_{\infty}<1
$$

$W_{\Delta V}$ and $W_{\Delta M}$ are respectively the VCM and MA uncertainty weighting functions, which must be selected by the designer. Based on the plant identification presented in Section 2, $W_{\Delta V}$ and $W_{\Delta M}$ were designed as first-order systems shown in Fig. 12.

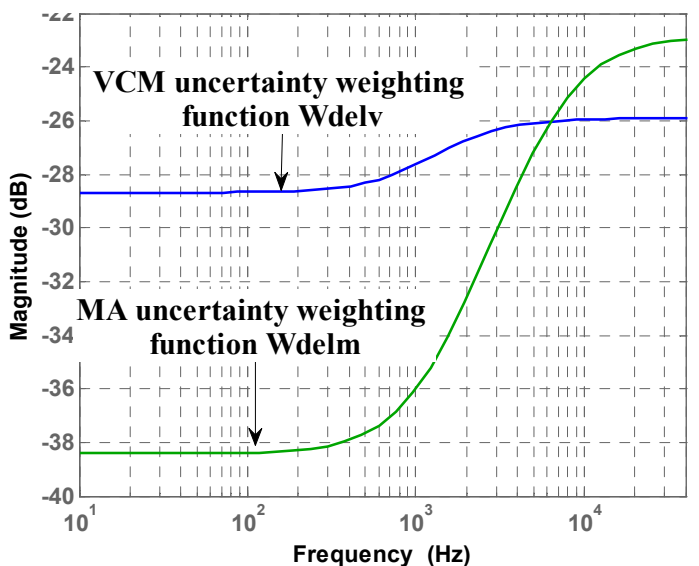

FIGURE 12. UNCERTAINTY WEIGHTING FUNCTIONS
The linear fractional transformation (LFT) for the mixed $\mathrm{H}_{2} / \mathrm{H}_{\infty}$ control design is shown in Fig. 13, in which the transfer function from $d_{\infty}$ to $z_{\infty}$ is used to measure the robust stability and $\gamma_{1}, \gamma_{2}$ and the control input weight $W_{u}$ are tuning parameters for the control synthesis. Then the optimal control synthesis can be written as the optimization problem in Eq. (5), where " $T_{A->B}$ " means the closed-loop transfer function from input " $A$ " to output " $B$ " and " $\|T\|_{2}$ " is the H-2 norm of the transfer function " $T$ ".

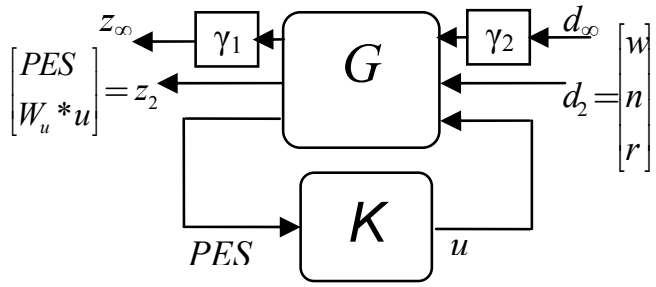

FIGURE 13. LFT FOR CONTROL SYNTHESIS

$$
\begin{aligned}
& \min _{K}\left\|T_{d_{2} \rightarrow z_{2}}\right\|_{2} \\
& \text { st. }\left\|T_{d_{\infty} \rightarrow z_{\infty}}\right\|_{\infty} \leq 1
\end{aligned}
$$

3.2.2 Control synthesis. To make the designed controller be more implementable, the mixed $\mathrm{H}_{2} / \mathrm{H}_{\infty}$ control was synthesized in discrete-time domain. There are several control synthesis methods [12] to solve Eq. (5). In this paper, the convex optimization approach via linear matrix inequalities [13] was utilized for the control synthesis. The closed loop system with the output $\left[z_{\infty}{ }^{T} z_{2}^{T}\right]^{\mathrm{T}}$ from the input $\left[d_{\infty}{ }^{T} d_{2}{ }^{T}\right]^{\mathrm{T}}$ can be written as:

$$
G_{c l}=\left[\begin{array}{c|cc}
A_{c l} & B_{c l \infty} & B_{c l 2} \\
\hline C_{c l \infty} & D_{c l \infty \infty} & D_{c l \infty 2} \\
C_{c l 2} & D_{c l 2 \infty} & D_{c l 22}
\end{array}\right]
$$

Then, by using the ideas of the congruent transformation, the optimization in Eq. (5) can be formulated as:

$\min \operatorname{trace}\{W\}$

$K, W, P_{2}, P_{\infty}$

$\begin{aligned} \text { st. } & {\left[\begin{array}{ccc}W & C_{c l 2} P_{2} & D_{c l 2} \\ * & P_{2} & 0 \\ * & * & I\end{array}\right] \succ 0,\left[\begin{array}{ccc}P_{2} & A_{c l} P_{2} & B_{c l 2} \\ * & P_{2} & 0 \\ * & * & I\end{array}\right] \succ 0 } \\ & {\left[\begin{array}{cccc}P_{\infty} & A_{c l} P_{\infty} & B_{c l \infty} & 0 \\ * & P_{\infty} & 0 & P_{\infty} C_{c l \infty}^{T} \\ * & * & I & D_{c l \infty}^{T} \\ * & * & * & I\end{array}\right] \succ 0 }\end{aligned}$ 
where the symbol "*" denotes the transpose of the corresponding element at its transposed position.

The equivalence between the two optimizations does not require $P_{2}=P_{\infty}$. However, it is necessary to impose the constraint

$$
P_{2}=P_{\infty}=P
$$

to recover the convexity of the mixed optimization [13]. The price of this restriction is that, as will be shown in the results that will be subsequently presented, a significant conservatism is thus brought into the design. As the parameters $\gamma_{1}$ and $\gamma_{2}$ are reduced to 0 , the solution of the mixed optimization $\mathrm{H}_{2} / \mathrm{H}_{\infty}$ optimization given by Eq. (7) and Eq. (8) converges to the nominal $\mathrm{H}_{2}$ design. However, with $\gamma_{1}=\gamma_{2}=1$ the solution of the mixed $\mathrm{H}_{2} / \mathrm{H}_{\infty}$ optimization given by Eq. (7) and the imposition of the constraint by Eq. (8) results in a very conservative control design, which attains a significant lower performance than that attained by the nominal $\mathrm{H}_{2}$ design which satisfies the robustness constraint imposed in Eq. (5). Moreover, reducing the control input weighting function $W_{u}$ to zero ("cheap" control), did not significantly alter the closed-loop sensitivity function from runout to $P E S$ of the mixed $\mathrm{H}_{2} / \mathrm{H}_{\infty}$ design, as shown in Fig. 14.

3.2.3 Nominal $\mathrm{H}_{2}$ control. In order to verify the inherent conservatism of the mixed $\mathrm{H}_{2} / \mathrm{H}_{\infty}$ control synthesis by using LMI optimization with Eq. (7) and Eq. (8), a nominal $\mathrm{H}_{2}$ controller was synthesized by tuning the input weighting value $W_{u}$ in order to satisfy the $\mathrm{H}_{\infty}$ constraint in Eq. (5). With the choice of $W_{u}=0.1 * I_{2}$, the designed controller can achieve the robust stability with $\left\|T_{d_{\infty} \rightarrow z_{\infty}}\right\|_{\infty}=0.941<1$. The resulting sensitivity function from runout to $P E S$ for the $\mathrm{H}_{2}$ control is shown in Fig. 14. As a consequence, we decided to implement the nominal $\mathrm{H}_{2}$ design in order to evaluate its performance on an actual drive.

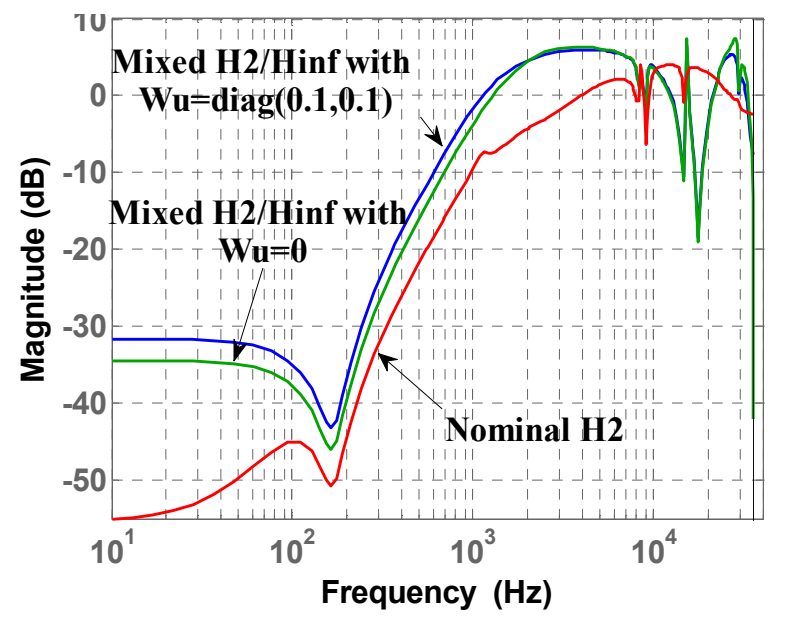

FIGURE 14. SENSITIVITY FUNCTIONS BY TUNING $W_{U}$
In order to investigate the benefit of the microactuator, the sensitivity function from runout to $P E S$ was decomposed into a product of a VCM sensitivity function and a MA sensitivity function, following the procedure in the sensitivity-decoupling design technique [4]. As shown in Fig. 15, the gain crossover frequency of the MA sensitivity transfer function is $5.48 \mathrm{KHz}$, which means the microactuator is significant for high frequency disturbance rejection.

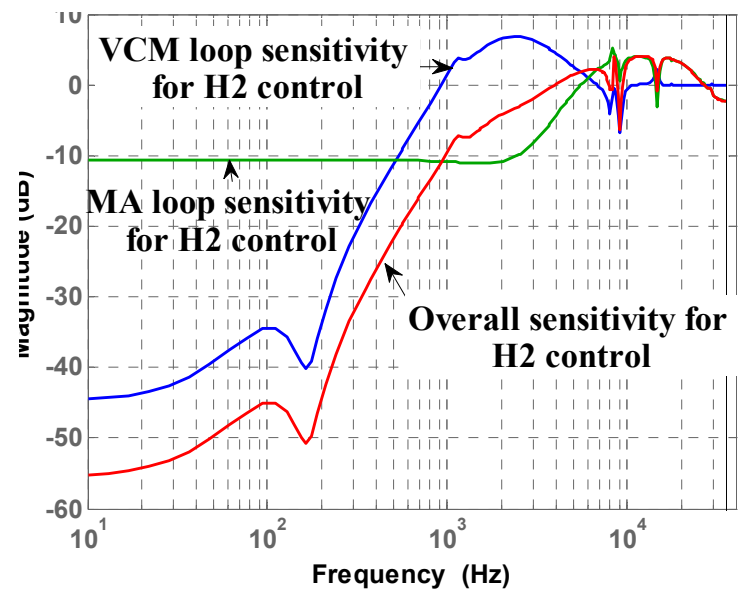

FIGURE 15. SENSITIVITY FUNCTION FOR $\mathrm{H}_{2}$ CONTROL

3.2.4 Add-on integral action. An integral action was added onto the designed controller to attenuate low frequency disturbances such as torque disturbances. Because of the small moving range of the PZT actuator, the integral action was only added onto the VCM controller as described in Eq. (9). In order to avoid degrading the performance of the original control, the parameters were chosen as $g=1, z_{0}=0.9972$. With the add-on integral action, the constraint for the nominal $\mathrm{H}_{2}$ control changes to $\left\|T_{d_{\infty} \rightarrow z_{\infty}}\right\|_{\infty}=0.939<1$.

$$
\tilde{K}(z)=\left[\begin{array}{cc}
g \frac{z-z_{0}}{z-1} & 0 \\
0 & 1
\end{array}\right] * K(z)
$$

\section{IMPLEMENTATION RESULTS}

\subsection{Simulated track runout}

In order to obtain a more realistic and complete evaluation of the dual-stage servo system performance, we used a computer generated runout signal to simulate track motions. The simulated track runout whose $3 \sigma$ value is $11.4 \mathrm{~nm}$, was generated from the power spectrum density of the real track runout data.

\subsection{Experimental results}

Firstly, in order to validate our system identification and control designs, the closed-loop sensitivity functions by using the sinusoidal sweeping signal were measured. Because of the conservatism of the mixed $\mathrm{H}_{2} / \mathrm{H}_{\infty}$ control, only the nominal $\mathrm{H}_{2}$ 
control with the better performance and the robust stability was implemented. The experiment results are shown in Fig. 16.

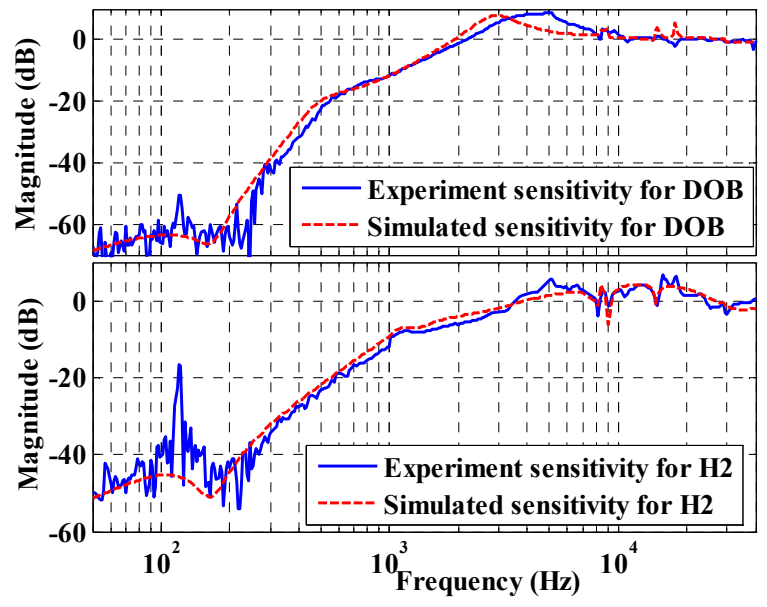

FIGURE 16. RESULTS OF SENSITIVITY FUNCTIONS

To analyze the closed-loop time-domain PES, 2048-point PES was recorded by the DSP. To highlight the advantage of the disturbance observer, the outer-loop sensitivity-decoupling controller was also implemented. The fast Fourier Transform (FFT) of the closed-loop PES without repeatable runout for the designed controllers is shown in Fig. 17.

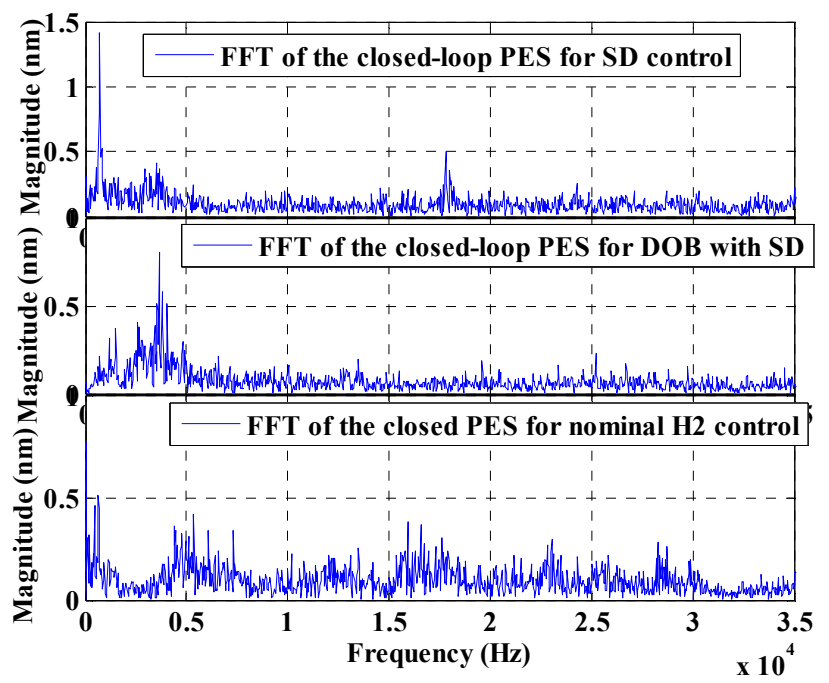

FIGURE 17. FFT OF THE CLOSED-LOOP PES

The designed controllers in this paper aim at rejecting non-repeatable runout, since repeatable runout can be cancelled very well by using some specific compensators, such as adaptive feedforward cancellation [2-3]. Therefore, it is helpful to take repeatable runout components out from the closed-loop PES to evaluate the designed controllers. Table 1 shows $3 \sigma$ PES values for the designed controllers with and without repeatable runout components respectively.
TABLE 1. IMPLEMENTATION RESULTS

\begin{tabular}{ccc}
\hline & $\begin{array}{c}3 \sigma \text { PES w/ } \\
\text { RRO }(\mathrm{nm})\end{array}$ & $\begin{array}{c}3 \sigma \text { PES w/o } \\
\text { RRO }(\mathrm{nm})\end{array}$ \\
\hline $\begin{array}{c}\text { Sensitivity } \\
\text { decoupling control }\end{array}$ & 18.1 & 17.0 \\
\hline Disturbance observer & 13.2 & 13.1 \\
\hline Nominal $\mathrm{H}_{2}$ control & 21.6 & 9.4 \\
\hline
\end{tabular}

\subsection{Discussion of experimental results}

For the areal density of $1 \mathrm{~Tb} / \mathrm{in}^{2}$, the track pitch is approximately $50 \mathrm{~nm}$, which requires that the $3 \sigma$ TMR budget should be approximately $5 \mathrm{~nm}$. In our experiments, the PZT-actuated suspension based dual-stage servo system achieved the best $3 \sigma$ PES of $9.4 \mathrm{~nm}$ without repeatable runout. However, there are several factors that may detrimentally affect the servo performance in our experimental setup, making our results too pessimistic.

First, the LDV resolution is $2 \mathrm{~nm}$, while the real PES resolution for the track pitch of $50 \mathrm{~nm}$ is around $0.2 \mathrm{~nm}$. In addition, the LDV measurement noise at low frequency caused by the low frequency drift is quite high and the RMS value of the measurement noise is of the order of magnitude of $100 \mathrm{~nm}$. Second, in our experiment one arm of the E-block was cut out, which may affect the original optimal structural dynamics of the E-block. Furthermore, we mounted the PZT-actuated suspension on the E-block by using a screw and E-POXY, which may introduce additional vibrations. Also, the disk drive we used in the experiment was modified from an off-the-shelf single stage commercial drive. Thus, the PZT-actuated suspension may not match the E-block very well and the slider flying height may not be set to its optimal value during the experiment.

\section{CONCLUSION}

The disturbance observer with the sensitivity-decoupling control and the mixed $\mathrm{H}_{2} / \mathrm{H}_{\infty}$ control with an add-on integral action were developed for the dual-stage track-following control. Because of the conservatism of the mixed $\mathrm{H}_{2} / \mathrm{H}_{\infty}$ control synthesis through LMIs, the nominal $\mathrm{H}_{2}$ control was designed by tuning control input weighting value to achieve robustness. Consequently, the designed nominal $\mathrm{H}_{2}$ control can achieve not only good performances but also the robust stability. The designs were experimentally validated using a PZT-actuated suspension based dual-stage servo system. The sensitivity-function crossover frequency of the disturbance observer design is $2.08 \mathrm{KHz}$ and that of the $\mathrm{H}_{2}$ design is 3.67 $\mathrm{KHz}$. The $3 \sigma$ PES without repeatable runout for the disturbance observer design is $13.1 \mathrm{~nm}$, while the $3 \sigma$ PES without repeatable runout for the $\mathrm{H}_{2}$ design is $9.4 \mathrm{~nm}$.

\section{ACKNOWLEDGMENTS}

The authors thank Western Digital Inc. and Hutchinson Technology Inc. with parts and valuable information to perform 
this study. This work was performed with funding partially provided a grant from the National Science Foundation (NSF) and support from the Information Storage Industry Consortium (INSIC) and UC Berkeley Computer Mechanics Laboratory (CML).

\section{REFERENCES}

[1] Y. Tomoyoshi, F. Masanori, S. Hiroyuki, T. Kazuhiko, 2006. "Servo technology writing technology". Fujitsu Scientific and Technical Journal, Vol. 42, n1, Storage Systems and HDD Technologies, pp. 93-102.

[2] A. Sacks, M. Bodson, and W. Messener, 1995. "Adaptive methods for repeatable runout compensation". IEEE Trans. On Magnetics, Vol. 31, No. 2, pp. 1031-1036.

[3] J. Zhang, R. Chen, G. Guo, and T.S. Low, 2000. "RRO compensation for dual stage servo system", IEEE Trans. Magnetics, September, pp. 3581-3584.

[4] Y. Li and R. Horowitz, 2001. "Mechatronics of electrostatic microactuators for computer disk drive dual-stage servo systems". IEEE/ASME Trans. Mechatronics, Vol. 6, pp. 988-992.

[5] S. Schroeck, and W. Messner, 1999. "Dual-stage servo conrtroller for HDD using MEMS actuator, IEEE Trans. On Magnetics, Vol. 35, pp. 2271-2273.

[6] R. Conway and R. Horowitz, 2008. "A quasi-newton algorithm for LQG controller design with variance constraints." Proc. of the Dynamic Systems and Control Conference, Ann Arbor, Michigan.

[7] T. Suzuki, T. Usui, M. Sasaki, F. Fujisawa, T. Yoshida, and H. Hirai, 1997. "MR-48 comparison of robust track-following control systems for a dual stage hard disk drive". Micromechatronics for Info. and Precision Equipment, Japan, July.

[8] X. Huang, R. Nagamune and R. Horowitz, 2006. "A comparison of multirate robust track-following control synthesis techniques for dual-stage and multi-sensing servo system in hard disk drives." IEEE Trans. on Magnetics, 42(7), July, pp. 1896-1904.

[9] T. Umeno and Y. Hori, 1991. "Robust Speed Control of DC Servomotors using Modern Two Degrees-of-Freedom Controller Design". IEEE Trans. on Industrial Electronics, Vol. 38, No. 5, Oct., pp. 363-368.

[10]H. Lee and M. Tomizuka, 1996. "Robust Digital Tracking Controllers for High-Accuracy Positioning Systems". IEEE Trans. on Industrial Electronics, Vol. 43, No. 1, Feb., pp. 48-55.

[11]R. Conway, 2008. "Multi-objective control design for discrete time periodic systems via convex optimization". Tech. Rep., University of California at Berkeley.
[12]X. Chen and K. Zhou, 2001. "Multiobjective $\mathrm{H}_{2} / \mathrm{H}_{\infty}$ Control Design". SIAM J. Control Optim, Vol. 40, No. 2, pp. 628-660.

[13]X. Huang, 2006. "Robust Track-Following Control Design for Dual-Stage Servo Systems with a MEMS Microactuator and an Instrumented Suspension". Ph.D. Thesis, University of California, Berkeley. 